\title{
An Investigation of Orientational Symmetry-Breaking Mechanisms in High Landau Levels
}

\author{
K. B. Cooper ${ }^{1}$, M. P. Lilly ${ }^{1}$, J. P. Eisenstein ${ }^{1}$, T. Jungwirth ${ }^{2,3}$, L. N. Pfeiffer ${ }^{4}$, and K. W. West ${ }^{4}$ \\ ${ }^{1}$ California Institute of Technology, Pasadena, CA 91125 \\ ${ }^{2}$ University of Texas, Austin, TX 78712 \\ ${ }^{3}$ Institute of Physics ASCR, Cukrovarnická 10, 162 53 Praha 6, Czech Republic \\ ${ }^{4}$ Bell Laboratories, Lucent Technologies, Murray Hill, NJ 07974
}

(October 24, 2018)

\begin{abstract}
The principal axes of the recently discovered anisotropic phases of $2 \mathrm{D}$ electron systems at high Landau level occupancy are consistently oriented relative to the crystal axes of the host semiconductor. The nature of the native rotational symmetry breaking field responsible for this preferential orientation remains unknown. Here we report on experiments designed to investigate the origin and magnitude of this symmetry breaking field. Our results suggest that neither micron-scale surface roughness features nor the precise symmetry of the quantum well potential confining the $2 \mathrm{D}$ system are important factors. By combining tilted field transport measurements with detailed self-consistent calculations we estimate that the native anisotropy energy, whatever its origin, is typically $\sim 1 \mathrm{mK}$ per electron.
\end{abstract}

A fundamental goal of contemporary condensed matter physics is to understand the ground state of the twodimensional electron system (2DES) and to explore its experimental signatures. In the presence of a large perpendicular magnetic field $B$, the kinetic energy of $2 \mathrm{D}$ electrons becomes quantized into discrete massively degenerate Landau levels (LLs). At high $B$ only the lowest $(N=0)$ LL is occupied, and the 2DES exhibits its most spectacular phenomenon: the fractional quantized Hall effect (FQHE) [1]. At lower magnetic fields the higher LLs become occupied, and in the third or higher $(N \geq 2)$ LL no FQHE states have been observed. Recent experiments have nevertheless uncovered extraordinary transport signatures unique to the high LL regime, pointing to the existence of a new class of many-body states distinct from the incompressible quantum fluids responsible for the FQHE [2,3]. The most striking of these signatures is the rapid development, at very low temperatures, of strong anisotropies in the longitudinal resistance of the $2 \mathrm{D}$ electron system.

Observed only in very high mobility samples and at temperatures below about $100 \mathrm{mK}$, the anisotropies in the longitudinal resistance are strongest near half-filling of the $N=2$ and several higher Landau levels. This corresponds to Landau level filling fractions $\nu$, defined as the ratio of the electron density $N_{s}$ to the degeneracy $e B / h$ of a single spin-resolved LL, of $\nu=9 / 2,11 / 2$, $13 / 2$, etc. For 2D electron systems in GaAs/AlGaAs heterostructures grown on [001]-oriented GaAs substrates, the anisotropies are consistently disposed so that the

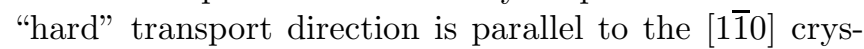
tallographic direction while the "easy" direction is parallel to [110]. Although a persuasive picture of how anisotropic electronic ground states develop at high Landau level occupancy now exists, there is still no understanding of why they are consistently oriented relative to the host crystal axes. The origin and magnitude of the necessary native symmetry breaking field is the focus of this paper.

The resistance anisotropy near half-filling of high LLs has been widely interpreted as evidence for chargedensity-wave (CDW) ground states. Hartree-Fock (HF) calculations [4, [5] have suggested that 2D electrons in half-filled high LLs form a unidirectional CDW, or "stripe" phase. At $\nu=9 / 2$, for example, the system is expected to phase separate into alternating regions of $\nu=4$ and $\nu=5$ with a period of about three times the cyclotron radius $R_{c}=\hbar k_{F} / e B$, or about $100 \mathrm{~nm}$ in typical samples. If these stripes are somehow preferentially oriented, anisotropy in the longitudinal resistance of the sample would likely result. Transport currents flowing perpendicular to such stripes would presumably encounter greater resistance than currents flowing parallel to them.

More recent theoretical work has generally supported the early HF predictions, albeit with some significant modifications. Numerical exact diagonalizations of small systems of electrons have uncovered sharp features in the susceptibility and structure factor of the 2DES in high LLs consistent with stripe formation at half filling [6]. Refinements to the HF approach suggest that the stripes are themselves unstable against modulations along their length [7,8]. The resulting "smectic crystal" would presumably be pinned and therefore insulating at very low temperatures. Quantum and/or thermal fluctuations are expected to melt the system and render it analogous to a nematic liquid crystal [9]. A nematic has translational but not rotational symmetry and might therefore exhibit anisotropic transport if a preferred direction exists. At still higher temperatures a nematic to isotropic transition is predicted to restore rotational symmetry [9, 10].

The consistency of the orientation of the transport anisotropy in high LLs has been established through experiments on a large number of GaAs/AlGaAs het- 
erostructure samples. Grown by molecular beam epitaxy (MBE), these modulation-doped 2D electron systems have low temperature mobilities typically around $10^{7} \mathrm{~cm}^{2} / \mathrm{V}$ s and densities ranging from $1.5-2.7 \times$ $10^{11} \mathrm{~cm}^{-2}$. The samples are usually illuminated briefly at low temperature with a red light emitting diode to enhance their transport characteristics but, as explained below, the existence and orientation of the high Landau level resistance anisotropy does not depend upon this. Although most measurements have been made using square samples whose sides are parallel to the [1̄̄0] and [110] directions, specific additional experiments have shown that the orientation of the transport anisotropy is insensitive to the geometry of the conducting region, at least in mm-sized samples [1].

In this paper we report new results which narrow down the list of possible native symmetry breaking effects that might orient the high LL anisotropy. Specifically, we show that neither the micron-scale morphology of the sample surface, as revealed by atomic force microscopy (AFM), nor the shape of the heterojunction potential confining the electron gas to two dimensions appear to be significant factors. In addition, by combining experimental results obtained using tilted magnetic fields with detailed numerical calculations, we estimate the strength of the still unknown symmetry breaking field.

Data from three samples (A, B, and C) will be presented here. Sample A consists of a single $30 \mathrm{~nm}$ GaAs quantum well embedded in thick layers of AlGaAs. Doping is provided by Si layers positioned symmetrically above and below the quantum well. Before illumination the 2DES in this sample has a density of $2.74 \times 10^{11} \mathrm{~cm}^{-2}$ and a low temperature mobility of $1.8 \times 10^{7} \mathrm{~cm}^{2} / \mathrm{V}$ s. Brief illumination at $1.6 \mathrm{~K}$ changes these values to $2.54 \times 10^{11} \mathrm{~cm}^{-2}$ and $2.1 \times 10^{7} \mathrm{~cm}^{2} / \mathrm{V} \mathrm{s}$, respectively. Samples B and $\mathrm{C}$ are conventional single heterointerfaces. After illumination these samples respectively have densities of 2.67 and $1.48 \times 10^{11} \mathrm{~cm}^{-2}$ and mobilities of 0.9 and $1.1 \times 10^{7} \mathrm{~cm}^{2} / \mathrm{V}$ s. Each sample was cleaved from its parent MBE wafer into a square, 3 or $5 \mathrm{~mm}$ on a side, and eight diffused In contacts were positioned at the corners and midpoints of the sides. Transport measurements were made with an excitation current of $I \leq 20 \mathrm{nA}$ at $13 \mathrm{~Hz}$ driven between midpoint contacts on opposite sides of the sample. Longitudinal resistance data were obtained by measuring the resulting voltage drop between corner contacts. Each of these samples clearly exhibits the characteristic low temperature resistance anisotropies near half-filling of the $N \geq 2$ LLs first reported by Lilly, et al. [2].

The inevitable imperfections which arise during crystal growth are natural candidates for an extrinsic mechanism capable of orienting the anisotropic electronic phases in high LLs. Since the kinetics of MBE growth is known to be anisotropic, it is easy to imagine that these imperfections might be preferentially oriented. This would break the rotational symmetry of the local environment of the 2DES and possibly influence its transport properties. As it is obviously difficult to directly observe the local environment of the $2 \mathrm{DES}$, which is buried inside the sample, we have resorted instead to an examination of the exposed top surface of the heterostructure via AFM [12]. Although this surface is typically $150 \mathrm{~nm}$ above the plane of the 2DES, the predicted wavelength of the CDW in high LLs is of comparable magnitude.

Nine high mobility GaAs heterostructure wafers (including those of samples A, B, and C) were examined in search of anisotropic surface morphologies. Each contained a 2 DES with mobility exceeding $0.8 \times 10^{7} \mathrm{~cm}^{2} / \mathrm{V} \mathrm{s}$ and density in the range of $1.5-2.7 \times 10^{11} \mathrm{~cm}^{-2}$. Unambiguous determinations of the $[1 \overline{1} 0]$ and $[110]$ directions were made using one or more techniques [13]. Samples from all nine wafers showed strong resistance anisotropies at $\nu=9 / 2,11 / 2$, etc. with the high resistance direction along $[1 \overline{1} 0]$.

Figure 1 shows the results of transport and AFM studies of samples A and B. The top two panels show longitudinal resistance data, taken at $T=50 \mathrm{mK}$, from the filling factor range $3<\nu<5$. Near $\nu=9 / 2$ the strong resistance anisotropy characteristic of half-filled high Landau levels is clearly evident. As the data show, the "hard" transport direction is along [1]0]. In contrast, the resistance near $\nu=7 / 2$ is nearly isotropic. This filling factor, which lies in the $N=1 \mathrm{LL}$, supports a weak fractional quantized Hall state which gives way to an anisotropic state only when a large magnetic field component parallel to the $2 \mathrm{D}$ plane is applied [11,22]. It is worth noting that the data from sample A shown here was obtained without illumination, verifying that the anisotropy effect does not depend upon this. Data from sample A after illumination will be discussed below.

The lower panels of Fig. 1 display $16 \times 16 \mu \mathrm{m}$ AFM images of the [001]-oriented surfaces of the wafers from which samples A and B were cleaved, and the [1피] and [110] directions are indicated by arrows. The images were obtained at room temperature using the "tapping mode" technique. In tapping mode, the microscope's cantilever tip oscillates at its resonant frequency as it is scanned across a surface. A feedback loop attempts to maintain the tip oscillation at a fixed amplitude by adjusting the average height of the tip above the sample surface; this average tip displacement is recorded as "height data." Transient changes in the oscillation amplitude, roughly a measure of the height derivative in the scan direction, are monitored as "amplitude data." The middle panels of Fig. 1 display height data, while the bottom panels display the corresponding amplitude data.

The inevitable slow drifts of the cantilever tip during image acquisition, while leaving the amplitude data unaffected, can obscure shallow surface features in the height data. To compensate for these drifts, the height images in the middle panels of Fig. 1 were subjected to a high- 

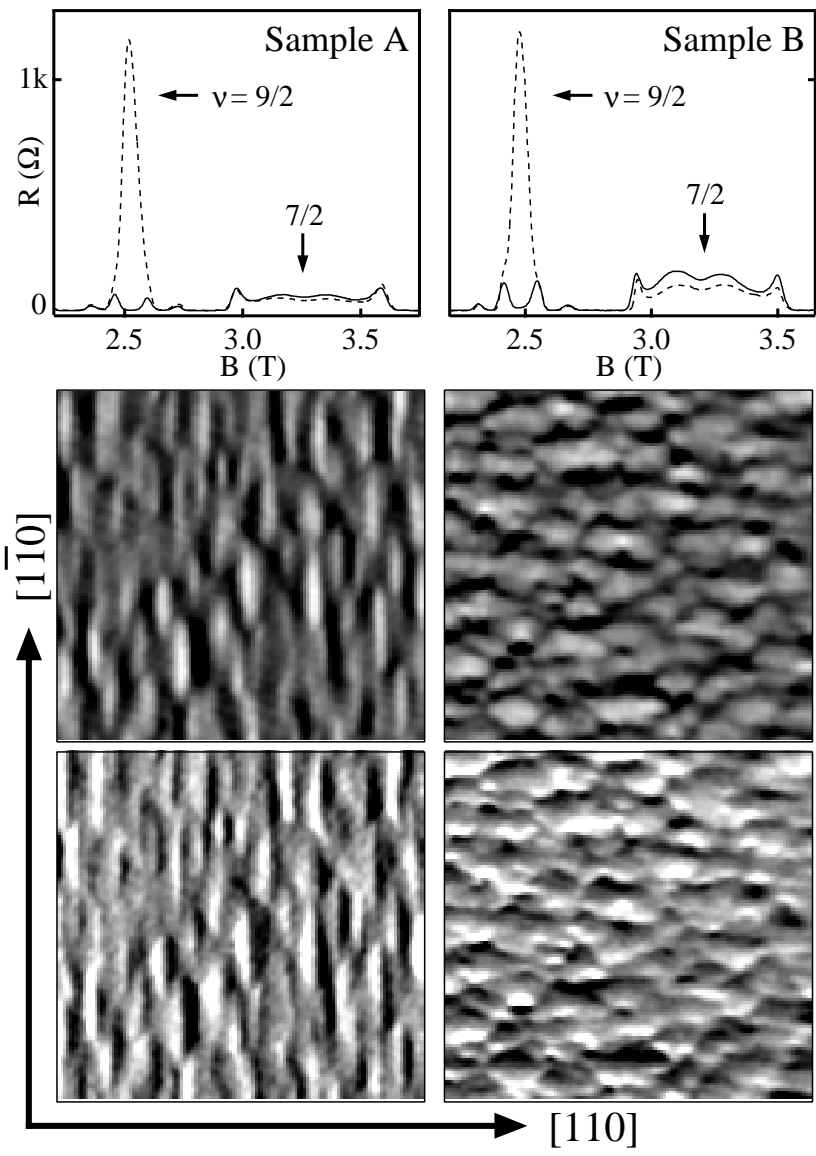

FIG. 1. Top panels: Longitudinal resistance data taken at $50 \mathrm{mK}$ in samples A and B. Dashed curves: resistance along [11̄0]; solid curves: resistance along [110]. Strongly anisotropic transport is evident around $\nu=9 / 2$. Middle panels: Height AFM images $(16 \times 16 \mu \mathrm{m})$ of the samples' parent wafers. The prominent mounds are $5-10 \mathrm{~nm}$ tall. Bottom panels: Corresponding amplitude AFM images. While for both samples the hard and easy transport directions at $\nu=9 / 2$ lie along [110] and [110], respectively, the orientations of their surface morphologies are perpendicular to one another.

pass filter in the scan direction. Because this filtering risks a loss of information of surface height variations perpendicular to the scan direction, four independent scans parallel to $[1 \overline{1} 0],[110],[010]$, and [100] were performed for each surface. These results (not shown here) confirm that the images of Fig. 1 faithfully represent all relevant morphology and that no spurious features related to the scan direction are present. Furthermore, the characteristic surface features revealed in Fig. 1 were found to cover each wafer's entire surface, indicating that their occurrence is a generic property of the MBE growth process.

Both surfaces in Fig. 1 exhibit shallow $(\leq 10 \mathrm{~nm})$, anisotropic surface features on the micron length scale. However, two important differences should be noted: first, the orientation of the surface features of the two samples are orthogonal to each other, and second, the general shapes of the surface features differ for the two samples. The surface of sample A, for example, is covered by long and straight cigar-shaped mounds oriented with their major axes along [110]. Sample B's surface, on the other hand, exhibits more irregularly-shaped mounds clearly elongated along [110] and separated by narrow, meandering valleys.

These results are typical of the variety of surface features seen on seven other wafers examined, where AFM images revealed varying degrees of mounding. Three of the seven showed mounds elongated along [110], two showed rounded mounds with virtually no preferred di-

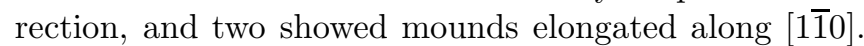
We emphasize, however, that in all the heterostructures the hard and easy axes of the resistance anisotropy in high LLs are along [1 $1 \overline{1} 0]$ and [110], respectively. Although the "quality" of the transport data does vary among the samples, it is sensibly correlated with the mobility of the 2DES and shows no evident dependence on the surface morphology.

The presence of anisotropic mounding, or roughness, on thin GaAs epilayers grown by MBE on [001]-oriented GaAs substrates has been noted previously by Orme, et al. 114. The competition between step-flow growth and island nucleation, in conjunction with anisotropic surface diffusion, was found to result in micron-sized mounds elongated along [11̄0]. Although Orme, et al. did not observe mounding oriented along [110], as we find in 4 of 9 samples studied, we note that our heterostructures are much thicker and more complex (consisting of hundreds of layers of GaAs and AlGaAs) than simple GaAs epilayers. As Orme, et al. found the tendency to mound to be very sensitive to growth temperature, substrate miscut angle, and epilayer thickness, the greater diversity of our results seems less surprising.

The lack of a consistent crystallographic orientation of the micron-scale surface roughness features on our samples contrasts sharply with the highly consistent alignment of the transport anisotropy axes exhibited by the $2 \mathrm{DES}$ at $\nu=9 / 2,11 / 2$, etc., in the same samples. From this we conclude that micron-scale surface roughness does not reflect the symmetry breaking field responsible for the alignment of the anisotropic electronic phases in high LLs. Whether finer scale surface features or unseen subsurface defects are involved remains to be determined. While we agree with Willett, et al. [15], that sufficiently severe surface roughness (whether as-grown or externally imposed) can influence 2DES transport, we do not find that the orientation of micron-scale surface features is always correlated with transport anisotropies in high LLs.

Another effect which might break the symmetry between the [110] and [110] directions in a 2DES on a [001]oriented surface is related to the shape of the potential well confining the electrons. Kroemer [16] has noted that 
while transport in bulk GaAs is invariant under $\pi / 2$ rotations about the [001] axis, this may not be the case in a 2DES. If the potential confining the electrons to the [001] plane lacks inversion symmetry the transport coefficients need only be invariant under $\pi$ rotations. While it seems unlikely that the band structure effects resulting from this lack of inversion symmetry could explain the large magnitude and sharp temperature and filling factor dependences of the high LL transport anisotropy, they may be sufficient to orient an otherwise spontaneously generated anisotropic ground state. Rosenow, et al. [17] and Takhtamirov, et al. [18] have stressed this point and have estimated that the reduced symmetry of typical single-interface heterojunctions leads to small $(\sim 0.1 \%)$ anisotropies in the $2 \mathrm{D}$ electron effective mass.

The transport measurements of samples A and B in Fig. 1 allow for examination of this idea. Sample A consists of a single $30 \mathrm{~nm}$ GaAs quantum well embedded within $\mathrm{Al}_{0.24} \mathrm{Ga}_{0.76}$ As. Silicon doping layers positioned $98 \mathrm{~nm}$ above and below the quantum well produce a symmetrically confined 2DES. Sample B, on the other hand, contains a single GaAs/AlGaAs interface doped from one side only. The 2DES in this sample is therefore confined by an asymmetric, roughly triangular, potential. In spite of this structural difference the two samples show qualitatively identical high LL transport features. In particular, the orientation of the anisotropy axes relative to the crystal axes is the same in the two samples. While one might argue that uncontrolled microscopic irregularities would destroy the symmetry of square quantum wells, it has been convincingly demonstrated, in 2D hole systems, that the symmetry of the confinement potential can have readily measurable transport consequences under appropriate circumstances 19,20]. Therefore, the lack of any significant differences in the high LL transport in samples A and B suggests that the symmetry of the confinement potential is also not a major factor in determining the orientation of the underlying anisotropic electronic states.

The transport data from sample A displayed in Fig. 1 , which were obtained without prior low temperature illumination, demonstrate that such illumination is not required to observe anisotropic transport in high Landau levels. Illumination is usually employed because it has been found empirically to often improve the quality of high field transport data. The precise way in which this occurs is not well understood, but in addition to typically increasing the $2 \mathrm{D}$ density and mobility, illumination also seems to improve the homogeneity of the electron gas. In any case, Fig. 2 demonstrates this improvement in sample A. The minima in the resistance measured along [110] at $\nu=9 / 2$ and $11 / 2$ are much deeper, the isotropic re-entrant integer quantized Hall states in the flanks of the Landau levels are much better developed [2,3,21], and additional structure is evident. Nonetheless, these data show clearly that illumination is not an important factor in the origin or orientation of the anisotropic phases of $2 \mathrm{D}$ electrons in high Landau levels.
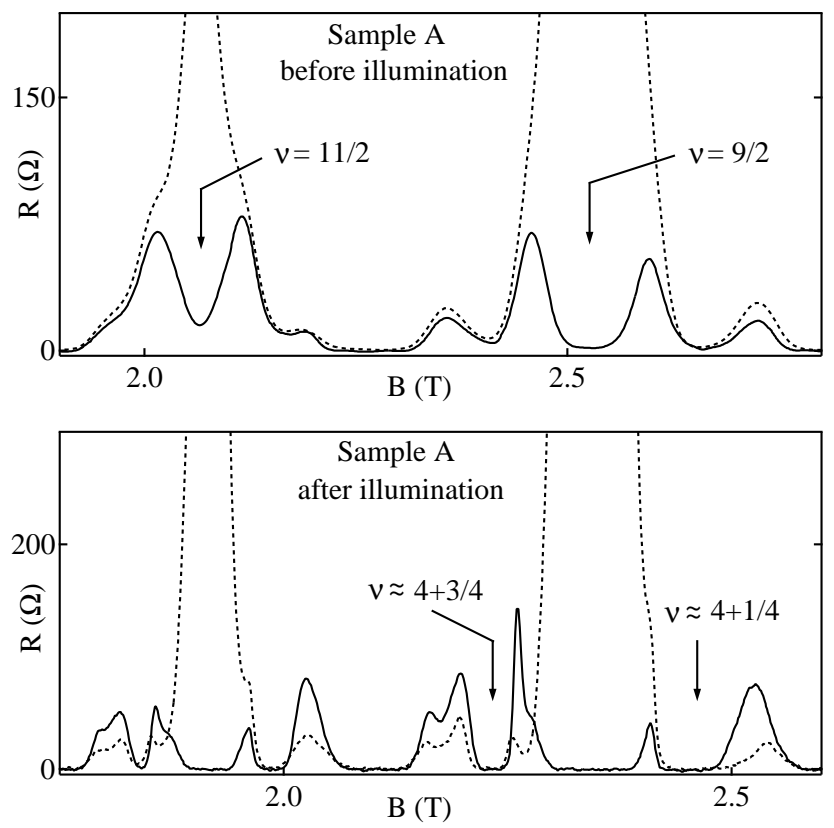

FIG. 2. Effect of low-temperature illumination by a red light emitting diode on the transport characteristics of sample A at $50 \mathrm{mK}$. Top: Longitudinal resistance along

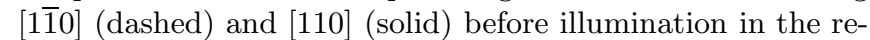
gion $4 \leq \nu \leq 6$. Bottom: The same measurement after illumination. Note the improvement in the degree of anisotropy at $1 / 2$ filling and the increased prominence of the re-entrant integer states near $1 / 4$ and $3 / 4$ filling.

A magnetic field component $B_{\|}$applied parallel to the plane of the 2DES provides an important tool for investigating the magnitude of the native symmetry breaking mechanism. Such an in-plane field allows the controlled introduction of an additional symmetry breaker which is known to be capable of altering the high Landau level transport anisotropy axes 11,22]. For example, only a relatively small $B_{\|}$pointed along [110] is required to interchange the original principal axes of anisotropy, with the new easy and hard directions along [110] and [110] respectively. Prior to this interchange, there is a special value of the in-plane field, $B_{\|}^{*}$, where the transport in the sample becomes approximately isotropic. Remarkably, if the in-plane field is instead directed along [1ํㅣ], the principal axes of anisotropic transport do not reverse.

Hartree-Fock calculations within the undirectional CDW model can account for these observations: the coupling between $B_{\|}$and the finite thickness of the 2D layer favors stripes aligned perpendicular to $B_{\|}$under typical circumstances 223,24. Assuming that the high resistance direction is perpendicular to the stripes, this conclusion is in agreement with experiment. Similar calculations 
have also been successful in explaining the more complex anisotropic transport behavior exhibited at high $B_{\|}$by quasi-2D systems in wide quantum wells having two occupied subbands [25]. These theoretical analyses also yield quantitative estimates of the field-induced anisotropy energy, $E_{A}$, defined as the difference in energy (per electron) of undirectional CDWs aligned parallel and perpendicular to the in-plane magnetic field. By evaluating the anisotropy energy at $B_{\|}^{*}$, these calculations provide an estimate of the magnitude of the native symmetry breaking field which orients the transport anisotropy in the absense of an in-plane field.

Figure 3 shows the effect of an in-plane magnetic field directed along [110] on transport in the vicinity of $\nu=9 / 2$ in samples A and C (data from sample B has been published previously [11). These data, which are obtained by tilting the sample relative to the axis of a single superconducting solenoid, clearly reveal the interchange of the anisotropy axes. For sample A a tilt of only $\theta=6^{\circ}$ is sufficient to render the transport roughly isotropic. This corresponds to $B_{\|}^{*} \approx 0.24 \mathrm{~T}$ at $\nu=9 / 2$. By $\theta=10^{\circ}$ the transport anisotropy is fully restored, albeit with interchanged axes. Samples $\mathrm{B}$ and $\mathrm{C}$ exhibit switching fields of approximately $B_{\|}^{*} \approx 0.50$ and $0.55 \mathrm{~T}$ respectively.
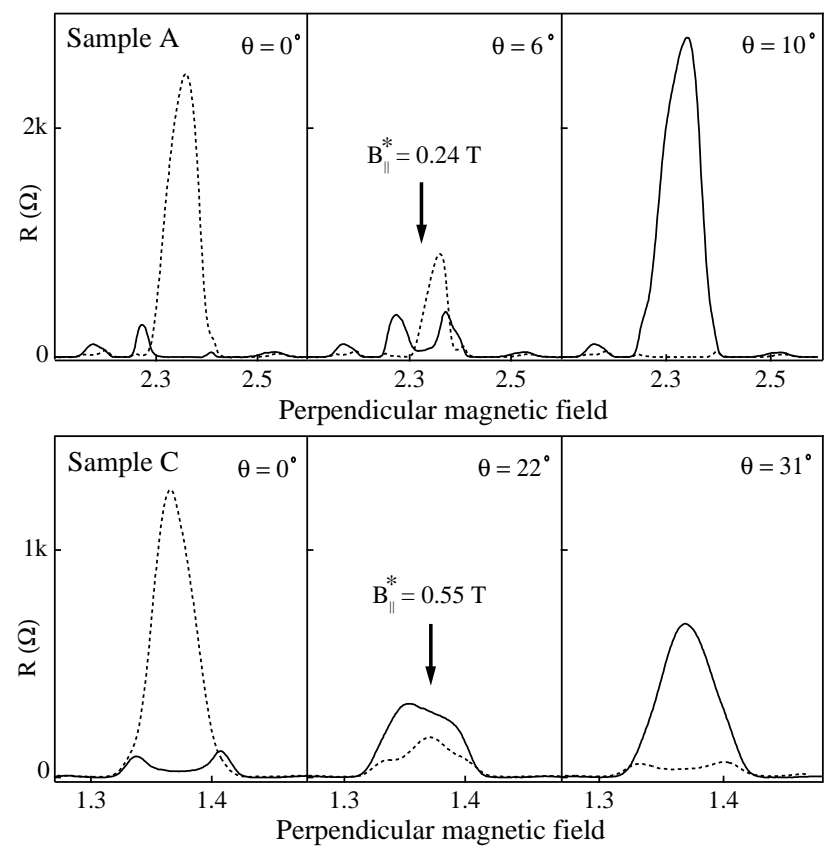

FIG. 3. Effect of a parallel magnetic field in the [110] direction on anisotropic transport at $\nu=9 / 2$ of samples $\mathrm{A}$ (50 $\mathrm{mK})$ and $\mathrm{C}(25 \mathrm{mK})$. Tilting about the [110] axis results in a reversal of the principal axes of anisotropy. Dashed: longitudinal resistance along [1 $\overline{1} 0]$. Solid: longitudinal resistance along [110].

Theoretical field-induced anisotropy energies are plot- ted in Figure 4. The many-body RPA/Hartree-Fock calculations are combined with a self-consistent localdensity-approximation description of one-particle states [23], obtained numerically for the specific structural parameters of samples A, B, and C. In agreement with the experimental observations, the unidirectional CDWs prefer being aligned perpendicular to the in-plane magnetic field direction $\left(E_{A} \geq 0\right)$. The agreement between experiment and theory on this point offers strong support to the overall CDW picture of the anisotropic phases in high Landau levels. After all, this theoretical result is not obvious a priori, as the interaction between a parallel magnetic field and the 2DES depends sensitively on the detailed finite-width profile of the 2DES and the nature of screening in high magnetic fields. Indeed, previous calculations have shown that unlike strongly confined $2 \mathrm{D}$ electron systems where the CDW orients perpendicular to $B_{\|}[23]$, in two-subband quantum wells the preferred CDW orientation is parallel to the in-plane field [25]. This observation helps explain the remarkable difference in the dependence of $E_{A}$ on $B_{\|}$in samples $\mathrm{A}$ and $\mathrm{B}$ despite the samples having nearly identical $2 \mathrm{D}$ electron densities. In sample B, the unoccupied second subband is much closer to the chemical potential than in sample A. Thus, being more similar to a system with two subbands occupied, a CDW in sample B should gain a smaller energy advantage by aligning perpendicular to a given in-plane field.

The calculated field-induced anisotropy energies at the measured $\nu=9 / 2$ switching fields $B_{\|}^{*}$ are $0.5,0.2$, and $2.4 \mathrm{mK}$ per electron in samples $\mathrm{A}, \mathrm{B}$, and $\mathrm{C}$ respectively. These energies are far smaller than the $\sim 100 \mathrm{mK}$ temperature scale that apparently governs the formation of the anisotropic electronic phase (i.e. the temperature at which transport becomes anisotropic). This disparity supports the model of a robust CDW oriented by a relatively weak native symmetry breaking field. Although the origin of the native symmetry breaking field remains unknown, these estimates of the anisotropy energy should offer useful constraints on proposed mechanisms.

The experimental results reported here suggest that two possible symmetry breaking effects, anisotropic surface roughness and confinement potential geometry, are not important factors in determining the orientation of the newly discovered anisotropic phases of 2D electrons in high Landau levels. Nevertheless, other possibilities remain. For example, Fil [26] has argued that the piezoelectricity of GaAs leads to lower energy for CDW's approximately aligned along either [110] or [110]. Although this does not explain what further distinguishes between these two directions, it does establish a mechanism which breaks rotational symmetry and favors directions which do have relevance to experiment.

In conclusion, we have addressed the still unresolved issue of how the anisotropic electronic states in high LLs become oriented relative to the crystallographic direc- 


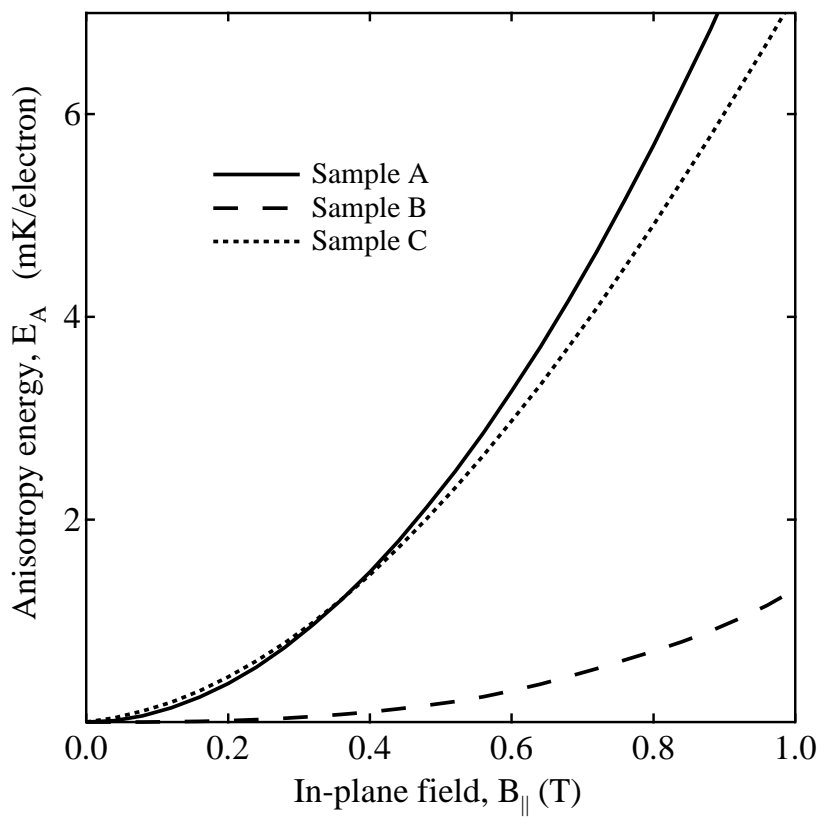

FIG. 4. Theoretical results of the anisotropy energy versus an in-plane magnetic field for a unidirectional CDW at $\nu=9 / 2$ in samples $\mathrm{A}, \mathrm{B}$, and $\mathrm{C}$. The CDW periods in the three samples, at $B_{\|}=0$, were calculated to be 108, 106, and $143 \mathrm{~nm}$ respectively. Evaluated at the switching field $B_{\|}^{*}$, the anisotropy energy yields an estimate of the native pinning energy of a CDW phase.

tions of the host semiconductor lattice. We have found that neither micron-scale surface roughness nor the symmetry of the confinement potential are major factors. In conjunction with detailed theoretical calculations, tiltedfield experiments support the overall charge density wave picture of the ground state of $2 \mathrm{D}$ electrons in high Landau levels and yield quantitative estimates of the native anisotropy energy for three different samples.

We thank M. Roukes for his generous loan of the atomic force microscope used in this work, E. Rashba for pointing out ref. 26 to us, R. Willett for showing us his data prior to publication, and S. Das Sarma and A.C. Gossard for helpful discussions. This work is supported by the NSF under grant DMR-0070890, the DOE under grant DE-FG03-99ER45766, and the Grant Agency of the Czech Republic under grant 202/01/0754.

* Present Address: Sandia National Laboratories, Albuquerque, NM 87185

[1] For recent reviews, see Perspectives in Quantum Hall Effects, edited by S. Das Sarma and A. Pinczuk, (John
Wiley, New York, 1997).

[2] M.P. Lilly, et al., Phys. Rev. Lett. 82, 394 (1999).

[3] R.R. Du, et al., Solid State Commun. 109, 389 (1999).

[4] A. A. Koulakov, M. M. Fogler and B. I. Shklovskii, Phys. Rev. Lett. 76, 499 (1996); and Phys. Rev. B 54, 1853 (1996). M. M. Fogler and A. A. Koulakov, Phys. Rev. B 55, 9326 (1997).

[5] R. Moessner and J. T. Chalker, Phys. Rev. B 54, 5006 (1996).

[6] E.H. Rezayi, F.D.M. Haldane, and K. Yang, Phys. Rev. Lett. 83, 1219 (1999).

[7] H. Fertig, Phys. Rev. Lett. Phys. Rev. Lett. 82, 3693 (1999) and H. Yi, H.A. Fertig and R. Cote, condmat/0003139

[8] A.H. MacDonald and M.P.A. Fisher, Phys. Rev. B61, 5724 (2000) and cond-mat/0001021.

[9] E. Fradkin and S. Kivelson, Phys. Rev. B59, 8065 (1999) and E. Fradkin, S. Kivelson, E. Manousakis, and K. Nho, Phys. Rev. Lett. 84, 1982 (2000).

[10] C. Wexler and A. Dorsey, cond-mat/0009096.

[11] M.P. Lilly, et al., Phys. Rev. Lett. 83, 824 (1999).

[12] AFM surface images were made with a Dimension 3100 Series Scanning Probe Microscope from Digital Instruments.

[13] The [110] and [1̄̄0] directions were distinguished via wetetch profiling, observation of "bowtie" defects on the sample surface, and knowledge of the parent wafer orientation. No conflicts arose.

[14] C. Orme, et al., Appl. Phys. Lett. 64, 860 (1994).

[15] R.L. Willett, et al., cond-mat/0007134.

[16] Herbert Kroemer, cond-mat/9901016.

[17] B. Rosenow and S. Scheidl, cond-mat/0003194.

[18] E.E. Takhmatirov and V.A. Volkov, cond-mat/0006226.

[19] J.P. Eisenstein, et al., Phys. Rev. Lett. 53, 2579 (1984).

[20] R. Winkler, et al., Phys. Rev. Lett. 84, 713 (2000).

[21] K.B. Cooper, et al., Phys. Rev. B60, R11285 (1999).

[22] W. Pan, et al., Phys. Rev. Lett. 83, 820 (1999).

[23] T. Jungwirth, A.H. MacDonald, L. Smrcka, and S.M. Girvin, Phys. Rev. B60, 15574 (1999).

[24] T. Stanescu, I. Martin, and P. Phillips, Phys. Rev. Lett. 84, 1288 (2000).

[25] W. Pan, et al., Phys. Rev. Lett. 85, 3257 (2000).

[26] D.V. Fil, Low Temp. Phys. 26, 581 (2000) and condmat/0004107 